\title{
A CSP Approach for Modeling the Hand Gestures of a Virtual Guitarist
}

\author{
Daniele Radicioni $^{1}$ and Vincenzo Lombardo ${ }^{1,2}$ \\ 1 Centro di Scienza Cognitiva, Università e Politecnico di Torino, \\ Via Po 14, 10123 Torino, Italy \\ 2 Dipartimento di Informatica, Università di Torino, \\ Corso Svizzera 185, 10149 Torino, Italy \\ \{radicion, vincenzo\}@di.unito.it
}

\begin{abstract}
This work presents a model for computing hand gestures of guitarists, within a broader system for the automatic performance of music scores. The fingering model encapsulates the main physical and bio-mechanical constraints that guitarists deal with in their daily practice, and is based on the CSP framework. It is interfaced with a physical model of the classical guitar, which uses the fingering to compute some sound synthesis parameters. We report on a preliminary test, where the fingerings computed by the model are compared with those provided by three human experts.
\end{abstract}

\section{Introduction}

In the last few decades there has been an increasing interest in exploiting AI techniques for computer music tasks, such as music analysis, composition, improvisation and performance, see e.g., [1], 9] and [5]. In the present work we tackle the task of music performance, that is the transformation of symbolic representations of a score into physical gestures, needed to operate a music instrument. An environment of music performance consists of the interpretation of the score, and the application of the gestures to some sound synthesis device that represents the instrument; gesture modeling is favorably coupled with a physical model of the instrument.

Within the broad area of the performer/instrument interaction, we emphasize the relevance of the fingering problem, which is an essential part of gesture modeling, deeply affecting the technical and expressive qualities of the sounds being produced. However, despite the salience of fingering in music performance, scores often lack of fingering indications, considered unnecessary (being common knowledge within a certain musical practice) or an execution choice. Namely, in the context of guitar performance, fingering defines for each note in the score both the position <string, fret> on the fingerboard, and the left hand finger involved in playing (thus yielding the triples <string, fret, finger>, called fingered positions), and sets the parameters that influence the final timbre of the sound during performance: the succession of the positions individuated is 
passed to a physical model of the classical guitar [2], which synthesizes the corresponding sounds. Provided that guitarists do use four fingers of the left hand (from the index to the little finger), $n$ notes generate up to $4^{n}$ different fingerings in the worst case. Since the same note can be found on up to 4 positions, this number might grow up to $16^{n}$. Thus, the case of guitar and of string instruments in general, is particularly challenging, and requires a considerable amount of experienced skills to human musicians, and automatic procedures aiming at an expressive performance outcome.

In this paper we illustrate a CSP approach for modeling the chord fingering process together with a preliminary experimental assessment of the result. CSP [3] is a well-known modeling technique for encoding problems expressed through constraints on variables; this approach is adequate for chord fingering, because the combination of fingered positions results from bio-mechanical and physical constraints over the hand and the instrument, respectively. This work is part of a larger project, that includes a bio-mechanical model for the hand gestures, estimating the major sources of difficulty in playing melodies [7, and adopts a graph-based search approach, that implements an overall motor behavior effortsaving strategy [8].

\section{Chord Fingering Modeled Via CSP}

Chord fingering is the counterpart in chronological terms of melody fingering, since while melodies are sets of notes to be played in sequence, chords are sets of notes to be played simultaneously. The fingering process takes in input chords described by a score in the traditional western music notation, and returns in output all the fingerings that satisfy a set of constraints coming from the instrument shape and the anatomy of the hand. The set of constraints is devised based on guitar handbooks from historical composers and contemporary teachers, and on a review of some of the didactic opuses of the early $19^{\text {th }}$ Century guitar composers, so to express which combinations of fingered positions could actually be played by a human performer 8. The fingering problem for chords can be cast in CSP terms as follows (Figure 1): we are given 1) a set of variables: the notes composing the chord; 2) a finite and discrete domain for each variable: from 1 up to 16 fingered positions per note; 3) a set of constraints defined over each pair of the original set of variables. The goal is to find one assignment to the variables such that the assignment satisfies all the constraints, thus being a viable fingering; yet, if more than a unique assignment arises, the goal is to rank them in order to predict which will be preferred by human performers.

The graph in Fig. 1 represents the chord fingering problem: each vertex represents a variable, so a note of the chord. The domain associated to that vertex includes all the fingered positions possible for that note. For example, the note $\mathrm{F} 2$, corresponding to the vertex $x$ in the graph, could be played on $\langle 6,1,1\rangle$, i.e., on the $6^{\text {th }}$ string, $1^{\text {st }}$ fret, by index finger; on $\langle 6,1,2\rangle$, by middle finger; and so forth. When two variables influence each other's domain, they are connected with an edge: this explains why the graph in Fig. 1] is completely connected, 


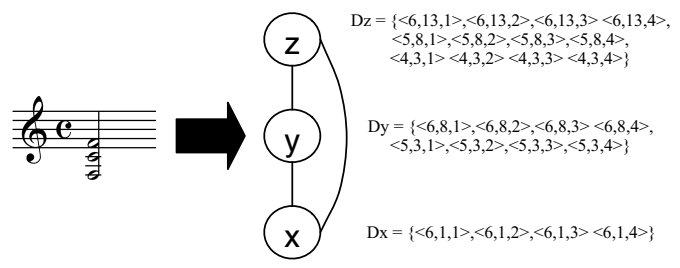

Fig. 1. A chord in input and the domains related to each note of the chord. Each triple in the note domains indicates <string, fret, finger>.

because all the fingered positions must be realized at the same time. The solution to the problem is computed by propagating the constraints to yield a simpler problem, and then performing a backtracking search to ground the variables.

Constraint propagation reduces the size of the variable domains while not affecting the final set of solutions. Since path-consistency has an exponential complexity, we adopt the directional arc-consistency, and directional path-consistency approaches [3, whose time and space complexity is $O\left(n^{3} k^{3}\right)$, where $n$ is the number of variables and $k$ bounds the domain sizes, being up to 16 . Recall that a graph is directional-arc-consistent relatively to order $d=\left(x_{1}, x_{2}, \ldots, x_{n}\right)$ iff every variable $x_{i}$ is arc-consistent relative to every variable $x_{j}$ s. t. $i \leq j$; and a graph is directional-path-consistent relatively to order $d=\left(x_{1}, x_{2}, \ldots, x_{n}\right)$ iff for every $k \geq i, j$ the pair $\left\{x_{i}, x_{j}\right\}$ is path-consistent relative to $x_{k}[3$. Directionality limits consistency to apply only along a given order on variables: in the case of directional path-consistency that is adopted in this paper, given the order $\left.<x_{1}, x_{2}, \ldots, x_{n}\right\rangle$ coming from the increasing pitch, the requirement is that for all $i, j \leq k$ we have that $\left\{x_{i}, x_{j}\right\}$ is path-consistent relatively to $x_{k}$.

Once the graph has been made directional path-consistent, depth-first search with backtracking occurs. The search follows the natural order, and starts by combining pairs of fingered positions from higher strings (namely, the basses), lower frets and lower fingers. Underpinned by the didactic guitar literature, the search strategy implements a preference for comfortable fingerings, given the evidence that, in absence of higher cognitive constraints, e.g. phrasing or punctuation, performers choose the bio-mechanically easiest solutions [4. This is immediately applicable to cases of chord fingering on spot (out of any musical context), like in pedagogical situations, which have been considered for the experimental validation.

\section{Preliminary Experiment and Conclusions}

To the ends of evaluating the model outlined, we compared its fingerings against those provided by three professional guitarists, to check $i$ ) whether the CSP approach, jointly with the set of physical and bio-mechanical constraints, allows computing the same hand gestures that actually guitarists adopt to play chords, and $i i$ ) whether the pruning strategy misses any solution given by the human 
performers. Both of these aspects are relevant, the former being concerned with the timbre reliability, and the latter with the system efficiency: the more predictive the system is, the more it can be pruningful, and then the more efficient. We selected 34 chords, all admitting at least two different fingerings; experts were requested to write on paper -when possible- three different fingerings for each chord, in the order of preference, with the triples <string, fret, finger>. They were also requested to indicate whether any fingering computed by the model was not practicable. The same chords were given in input to the model, and the output annotated.

All the fingerings computed by the model have been found viable $(100 \%$ precision); the fingerings provided by human experts are a subset of the ones computed by the model, except for overall 6 cases (over 218 fingerings computed in total by the model, so $2.75 \%$ missing $-97.25 \%$ recall). Moreover, if we neglect the finger component of the triple, in the $92.1 \%$ of the cases the model correctly predicts the preferred fingering indicated by experts (which, disregarding the finger involved, agree in the $97 \%$ of the cases). Similarly, if we restrict the comparison between experts and model to the three cases where the experts exactly agreed on the triplets, the success ratio of the model is $75 \%$.

Several complexity factors were disregarded, which may suggest a guideline for future work, such the fact that chords might also be part of musical patterns together with melodic fragments, and such patterns may be learned, stored and retrieved as a block [6]. Future work will attempt accounting for the fingering of whole blocks of notes, where the case-based reasoning methodology would seem to be profitable: such improvements will lead us to consider real pieces from the classical repertoire, and to explore the automatic expressive performance, where the modeling of instrument/performer interactions plays a central role.

\section{References}

1. Balaban, M., Ebcioglu, K., Laske, O. (Eds): Understanding Music with AI Perspectives on Music Cognition, Menlo Park/CA: The AAAI Press, 1992.

2. Cuzzucoli, G., Lombardo, V.: Physical model of the played classical guitar, including the player's touch, Comput. Music J., 23, 52-69, Summer 1999.

3. Dechter, R.: Constraint processing, Morgan Kaufmann, San Francisco/CA, 2003.

4. Heijink, H., Meulenbroek, R.G.J.: On the Complexity of Classical Guitar Playing: Functional Adaptations to Task Constraints, J. Motor Behav., 34, 339-351, 2002.

5. Lopez de Mantaras, R., Arcos, J.L.: AI and Music: From Composition to Expressive Performance, AI Mag., 23, 43-57, 2002.

6. Parncutt, R., Sloboda, J., Clarke, E., Raekallio, M., Desain, P.: An ergonomic model of keyboard fingering for melodic fragments, Music Percept., 14, 341-382, 1997.

7. Radicioni, D., Anselma, L., Lombardo, V.: A prototype to compute string instruments fingering, Proceedings of the Conference on Interdisciplinary Musicology (CIM04), Graz/Austria, 2004, http://gewi.uni-graz.at/ cim04/.

8. Radicioni, D., Lombardo, V.: Guitar fingering for music performance, Proceedings of the International Computer Music Conference, Barcelona/Spain, 2005.

9. Widmer G.: Discovering Simple Rules in Complex Data: A Meta-learning Algorithm and some surprising musical discoveries, Artif. Intell., 146, 129-148, 2001. 